\title{
Towards Automated Dependability Analysis of Dynamically Connected Systems
}

\author{
Paolo Masci, Marco Martinucci and Felicita Di Giandomenico \\ Information Science and Technologies Institute \\ Italian National Research Council - Pisa, Italy \\ e-mail: \{masci,martinucci, digiandomenico\}@isti.cnrit
}

\begin{abstract}
Dynamic environments may include autonomous and decentralised components that pose many challenges from the point of view of interoperability, thus triggering research studies in several directions. One recent research direction explores the automatic composition of heterogeneous systems through connectors synthesised at run-time. Besides functional properties, such connectors generally need to satisfy also non-functional (dependability-related) properties. This paper investigates the definition of an automated procedure to support the synthesis of dependable connectors.
\end{abstract}

Keywords-Stochastic modelling, autonomous systems interoperation, automated dependability analysis.

\section{INTRODUCTION}

Ubiquitous computing refers to a vision of technology where devices are seamlessly integrated in the environment and everyone benefits from their services without even being aware of their presence [1]. In such a vision, complex and pervasive systems are expected to be composed of autonomous and decentralised components that cooperate and require services on the basis of specific needs. Pervasive systems may evolve along time, rely on heterogeneous communication protocols, and dynamically establish communication at run-time. The fast pace at which technology evolves continuously undermines the effectiveness of such systems because ubiquitous systems components need to exchange information and this ability is generally linked to the level of interoperability of their underlying technologies.

Among the current studies addressing the issue of interoperability of the next generation systems, the European Project ConNeCT [2] targets the dynamic synthesis of connectors via which networked systems communicate. The resulting emergent connectors compose and adapt interaction protocols run by the networked systems. The dynamic synthesis of connectors relies on activities related to discovery, learning, dependability [3] and performance analysis: interaction protocols are, in general, not known a priori, and both functional and non-functional properties must be taken into account in order to synthesise a proper connector.

In this work, we report on the research we are undertaking towards the development of a Dependability unit that implements an automated dependability and performance analysis to support the synthesis of connectors. The unit employs a stochastic model-based analysis, which shows appropriate to support design decisions during connector synthesis. In the remainder of the paper, like in the title, we will use the term "dependability" as a label that includes both dependability and performance attributes.

The paper is organised as follows. Section II summarises the main aspects of our reference framework, which includes the units for Discovery/Learning, Synthesis, Dependability, and Monitoring. The architectural structure of the Dependability unit is introduced in Section III. A case study on a distributed and heterogeneous marketplace application is presented in Section IV. The final considerations and conclusions are drawn in Sections V and VI.

\section{REFERENCE FRAMEWORK}

Following the approach taken in the CONNECT Project, which is general and well suited for addressing interoperability in the context of complex pervasive systems, we consider a reference framework with four logical units: Discovery/Learning, Synthesis, Dependability and Monitoring.

Discovery/Learning: This unit gathers information on the networked systems. Specifically, the unit discovers mutually interested networked systems, and retrieves information on the specification of the interfaces of the networked systems. The unit assumes that networked systems are discovery enabled, i.e., they provide a minimal description of their intent and functionalities. In the case a partial behaviour description is provided, the unit completes the specification through a learning procedure (e.g., via model-based testing).

Synthesis: This unit performs the dynamic synthesis of mediating connectors to enable interoperation among networked systems willing to interact. The unit uses the behavioural models built by Discovery/Learning to identify mismatches between the communication protocols employed by the networked systems, generates a connector that resolves such incompatibilities and deploys the connector.

Dependability: This unit supports the Synthesis unit in the definition of a connector that enables networked systems to interact with a given dependability level. Specifically, the unit executes a stochastic model-based quantitative analysis to determine if the connected system can meet the required dependability level. Furthermore, in the case the requirements are not satisfied, the Dependability unit reports possible enhancements to Synthesis.

Monitoring: This unit becomes operational when the connector is deployed. The unit continuously monitors the 


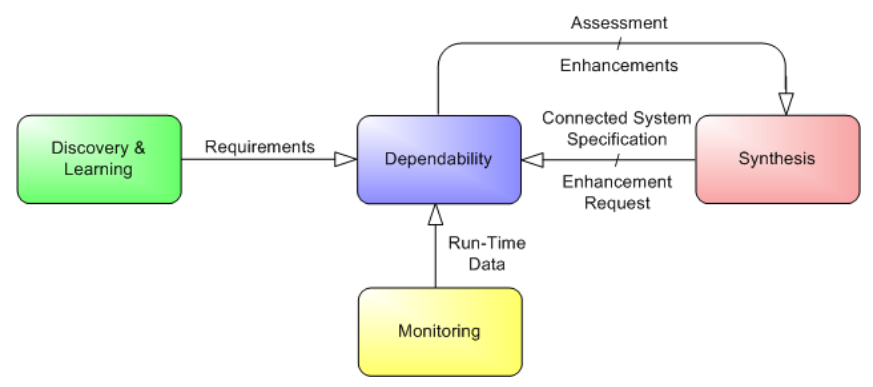

Figure 1. Input-Output relations of the Dependability unit.

connector to update the functional and non-functional specification of the connector with run-time data.

In the Connect framework, a networked system broadcasts a connect request whenever a new connection to a service is needed. The connect request contains a description of the requested service together with a specification of the required dependability level for the service. When Discovery/Learning detects a connect request, the unit searches the networked systems that can provide the requested service. If such systems are found and operate a communication protocol different from that of the networked system that made the connect request, Discovery/Learning activates Synthesis to generate a suitable connector that enables interoperation. The Synthesis unit, on the basis of the specification of the communication protocols, produces a mediating connector. Before connector deployment, Synthesis activates the Dependability unit to evaluate if the connected system that will be obtained satisfies the non-functional requirements expressed by the networked systems. Once the connector is deployed, its run-time behaviour is observed by the Monitoring unit and notified to other units according to their needs, to properly account for dynamic system changes.

In this work, we elaborate on the Dependability unit. A Dependability-centric view of the input-output relations between the Dependability unit and the other units is shown in Figure 1: Discovery/Learning provides the dependability requirements; Synthesis provides the specification of the connected system, and possibly requests a dependability enhancement; Monitoring provides run-time statistical data on the execution of the deployed connector. The dependability assessment and the enhancements determined by the Dependability unit are used by Synthesis.

\section{ARChitecture of the Dependability Unit}

The Dependability unit is logically split into four main functional modules (see Figure 2): Builder, Analyser, Evaluator and Enhancer. The Builder module derives the dependability model of the CONNECTed system from the specification provided by Synthesis. The Analyser module uses the generated dependability model to perform a quantitative assessment of the non-functional requirements reported by the Discovery Unit. The Evaluator module checks the analysis results to determine if the non-functional

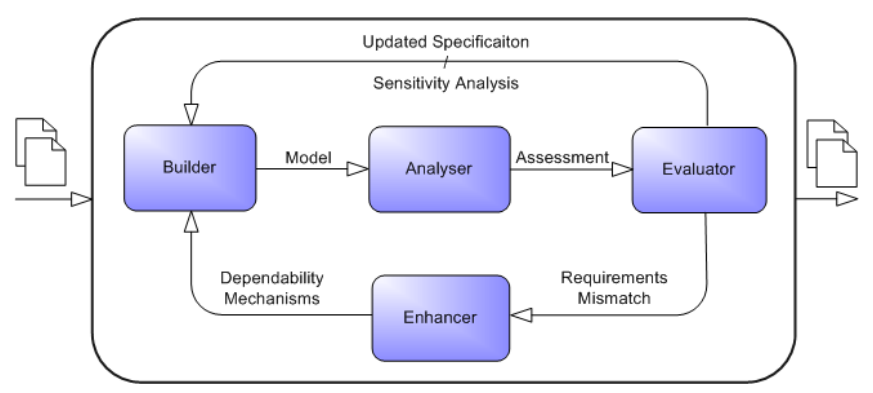

Figure 2. Architecture of the Dependability unit.

requirements are met. If the requirements are not satisfied, the Evaluator activates the Enhancer module to determine possible solutions to improve the dependability level of the CONNECTed system, e.g., use an updated specification that takes into account an alternative connector deployment or enhance the connector specification with ad hoc dependability mechanisms. If the requirements are satisfied, the Evaluator reports to Synthesis that the CONNECTor can be successfully deployed, and reports to the Monitoring Unit the aspects that must be observed for the CONNECTor that is going to be deployed, e.g., transition durations and probability of transitions failure.

In the following, we provide more details on the functionalities of the modules of the Dependability unit, and report on a prototype implementation, denominated DEA (DEpendability Analyser) of the Dependability unit based on the Möbius [4] analysis tool.

\section{A. Builder}

The Builder module takes in input the specification of the connected system from Synthesis, and the dependability requirements from Discovery/Learning. The specification includes the nominal behaviour of the connected system, i.e., in fault-free situations, and the exceptional conditions, i.e., the failure modes. The module produces in output a dependability model of the connected system that contains enough details to assess the given dependability requirements.

Specification of the connected system: With reference to recent works on synthesis of mediating connectors [5] and automata discovery/learning [6], the specification of the connected system is given with Labelled Transition Systems (LTSs) annotated with non-functional information necessary to build the dependability model of the connected system. An LTS is an abstract machine that represents the sequence of actions performed by the system. Formally, an LTS is a tuple $\left(\mathcal{S}, \mathcal{S}_{0}, \mathcal{L}, \mathcal{T}\right)$, where $\mathcal{S}$ is a set of states, $\mathcal{S}_{0} \in \mathcal{S}$ is a set of initial states, $\mathcal{L}$ is a set of labels, and $\mathcal{T} \subseteq \mathcal{S} \times \mathcal{L} \times \mathcal{S}$ is a transition relation. Annotations include, for each labelled transition, the following fields: time to complete, firing probability, failure mode, and failure probability.

Dependability requirements: Typical requirements that need to be satisfied are related with availability, reliability, performance and their combination. In our architecture, the 
dependability requirements provided by the networked systems are translated by Discovery/Learning into metrics and guarantees. Metrics are arithmetic expressions that describe how to obtain a quantitative assessment of the properties of interest of the connected system. In this context, metrics are expressed in terms of transitions and states of the LTS specification of the networked systems. Guarantees are boolean expressions that are required to be satisfied on the metrics under a set of constraints.

Dependability model: The dependability model of the connected system is specified with a formalism that allows to describe complex systems that have probabilistic behaviour, e.g., stochastic processes.

Implementation: The prototype implementation of the Builder module takes in input the LTS of the connected system described with Finite State Processes (FSP) [7]. The dependability model of the system is specified with Stochastic Activity Networks (SANs), a generalisation of Stochastic Petri Nets introduced in [8] and formally defined in [9]. The SAN model is obtained from the LTS model by using the theory of regions [10]. A region identifies a set of states in the LTS such that all transitions with the same label either enter, exit, or never cross the boundary of the region. Each region in the LTS corresponds to a place in the derived SAN model, and each labelled transition in the LTS corresponds to an activity in the SAN model. A similar approach has already been used in other works to translate LTSs into Petri Nets (see, for instance, [11], [12] and [13]). In our approach, in order to have a well-defined probabilistic model, non-deterministic choices among $k$ transitions outgoing from an LTS state are mapped in the SAN model into instantaneous activities with $k$ case probabilities. The metric is an arithmetic expression that may contain a predefined set of functions (see Table I for some examples). The guarantee is given by a boolean expression on the metric and a set of constraints on the connected system model (e.g., constraints on the time frame of evaluation of the metric and constraints on the behaviour of the networked systems). Statistical operators (e.g., mean and variance), comparison and logical operators can be used in the expression. In order to uniquely identify states and transitions of the metric expression that appear in the generated SAN model, renaming is used to constrain the definition of the LTS regions.

1) Analyser: The Analyser module takes in input the dependability model from the Builder module and the dependability requirements from Discovery/Learning. The module builds a reward model, i.e., a model that enables a quantitative assessment of the metrics of interest, and makes use of a solver engine to obtain a quantitative assessment of the dependability metrics.

Reward model: The reward model is the dependability model extended with reward functions. Reward functions allow to specify properties of interest: they return a value depending on the system state, and can be evaluated either at an instant of time or accumulated over a time frame.

Solver: The solver evaluates the reward functions defined in the reward model. The evaluation can be performed either with analytical approaches or with simulation. Analytical approaches are based on state space analysis, and they are applicable when the model does not face state space explosion and when timing information follow deterministic or exponential distributions. Simulation, on the other hand, is always applicable (unless extremely rare events have to be evaluated), and is expected to be particularly useful when a tradeoff between accuracy and time to perform the assessment is needed.

Implementation: The prototype implementation of the Analyser is based on Möbius [4], a popular software tool that provides a comprehensive framework for model-based evaluation of dependability and performance aspects of systems. The framework supports, among others, the SAN formalism. In Möbius, each reward function is a $\mathrm{C}++$ function that returns a value depending on the marking of the SAN. There are two kinds of reward functions: rate rewards and impulse rewards. Rate rewards are used to implement time-based reward functions. Impulse rewards are used to implement action-based reward functions, i.e., they are associated with specific activities and can be evaluated only when the associated activity completes. The reward functions are automatically derived from the metrics expression as follows: the metric is mapped into its syntax tree to decompose the metric into a combination of basic functions; the basic functions are translated into $\mathrm{C}++$ functions by using a predefined repository of function templates. For instance, with reference to the functions shown in Table I, a rate reward template is used to translate timeFrame $(s)$, while an impulse reward template is used to translate \# $(t r, t 1, t 2)$. The content of the repository of function templates is not shown in this work, but some details will be provided in the case study shown in Section IV. The Solver evaluates the reward functions via simulation. The quantitative assessment of the metric is obtained from the assessment of the reward functions by merging the results according to the arithmetic operations specified in the syntax tree of the metric expression.

\section{B. Evaluator}

The Evaluator reports to Synthesis if the connected system satisfies the dependability requirements provided by Discovery/Learning. In the case of requirements mismatch, the Evaluator sends a warning message to Synthesis, and may receive a request to evaluate if enhancements can be applied to improve the dependability level of the connected system.

Requirements mismatch: If the requirements are not satisfied, the Evaluator may receive a request to explore one of the following three directions for improvements.

(1.) Update the specification of the connector to take into account an alternative connector deployment (e.g., a 


\begin{tabular}{ll}
\hline Function & Description \\
\hline timeFrame $(s): \mathcal{S} \rightarrow \mathbb{R}^{+}$ & returns the interval of time when the system is in state $s$ \\
minTimeStamp $(\operatorname{tr}): \mathcal{T} \rightarrow \mathbb{R}^{+}$ & returns the first instant of time when transition $t r$ fires \\
avgTimeStamp $(\operatorname{tr}): \mathcal{T} \rightarrow \mathbb{R}^{+}$ & returns the average instant of time when transition $t r$ fires \\
maxTimeStamp $(\operatorname{tr}): \mathcal{T} \rightarrow \mathbb{R}^{+}$ & returns the last instant of time when transition $t r$ fires \\
$\#(\operatorname{tr}, t 1, t 2): \mathcal{T} \times \mathbb{R}^{+} \times \mathbb{R}^{+} \rightarrow \mathbb{N}$ & returns the number of times transition $t r$ fires during the time frame $[t 1, t 2]$ \\
$\#(l, t 1, t 2): \mathcal{L} \times \mathbb{R}^{+} \times \mathbb{R}^{+} \rightarrow \mathbb{N}$ & returns the number of times transitions with label $l$ fire during the time frame $[t 1, t 2]$ \\
\hline
\end{tabular}

Table I

EXAMPLES OF PREDEFINED FUNCTIONS THAT CAN BE USED IN THE METRIC EXPRESSION.

deployment with lower failure rate). Upon receiving this request, the Evaluator triggers a new analysis that considers the updated specification of the connector.

(2.) Enhance the specification of the connector by including dependability mechanisms (e.g., a message retransmission technique). Upon receiving this request, the Evaluator triggers a sensitivity analysis whose objective is to understand which failure modes of the connected system have highest impact on the dependability measure. Such failure modes will be the first ones to be considered for devising appropriate counter-measures capable of limiting the failure effects. To this end, the Evaluator builds a sensitivity analysis campaign to instruct the Builder module on the creation of dependability model variants, each of which considers a specific subset of failure modes (among those foreseen). Whenever a variant is generated, the Analyser module performs the assessment of the metrics on the generated model. The Evaluator collects the analysis results and, after all variants have been analysed, produces a ranking of the failure modes. This ranking is used by the Evaluator to iteratively activate the Enhancer module as long as either the guarantees are satisfied, or the Enhancer signals that all possible dependability mechanisms have been explored.

(3.) Apply a combination of the previously mentioned enhancements.

Implementation: The Evaluator compares the analysis results against the guarantees specified in the requirements, and reports a message containing a boolean value to Synthesis. The Evaluator may receive from Synthesis the following three types of enhancement requests: alternative deployment: upon receiving this request, the Evaluator triggers a new analysis that considers the updated annotated LTS specification of the connector contained in the request.

dependability mechanism: upon receiving this request, the Evaluator triggers a sensitivity analysis that considers the impact of one failure mode at a time. Specifically, for each failure mode, a variant of the SAN models is generated such that the considered failure mode is the only one considered in the model. The ranking of the failure modes is obtained by assessing the metrics on the generated variants: the higher the impact on the guarantees dissatisfaction, the higher the ranking of the failure mode.

combined enhancement: a request that allows a combined use of the previously mentioned enhancements. The combined enhancement request contains the annotated LTS spec- ification of the connector for the alternative deployment, and an indication that the Evaluator module should also enable the evaluation of dependability mechanisms to improve the connector. A field in the request is used to specify if the enhancements should be evaluated individually and/or in combination. When evaluated individually, an evaluation ordering is also provided.

\section{Enhancer}

The Enhancer is activated by the Evaluator when the guarantees are not satisfied and Synthesis makes a request to enhance the connector with dependability mechanisms. The Enhancer is instructed by the Evaluator module on the requirements mismatch and the failure mode that needs to be tackled. Specifically, the Enhancer performs the following actions: (i) selects a dependability mechanism that can be employed, among those available, to contrast the failure mode indicated by the Evaluator module; (ii) if all possible applications of dependability mechanisms have already been explored without success, the Enhancer sends a warning message to the Evaluator module; otherwise, the Enhancer instructs the Builder module on the application of the selected dependability mechanism in the connected system model and triggers a new analysis.

Dependability mechanisms: Dependability mechanisms are counter-measures that can be adopted to contrast failure modes. Typically, dependability mechanisms are based on the application of redundancy, e.g., duplication of system channels, or retry of message transmissions over system channels. The dependability mechanism, in this context, will be embedded in the synthesised connector, because networked systems are not under the control of the framework. Nevertheless, the dependability mechanisms embedded in the connector can be employed to improve, to some extent, the dependability level of the networked systems. For example, the reliability level of a transmission performed by a networked system can be improved through timeouts or message retransmissions applied at the connector level.

Implementation: The dependability mechanism suitable to contrast a given failure mode is determined through an ontology of dependability mechanisms, such as that reported in [14]. A detailed discussion on the content of the ontology is out of the scope of this paper. We developed ad hoc dependability models for a set of relevant dependability mechanisms, and a set of rules to automate the application 


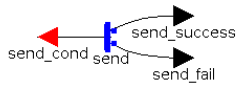

(a) Basic model.

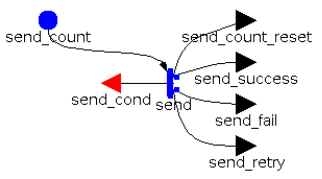

(b) Enhanced model.
Figure 3. Example of dependability mechanism and application rule.

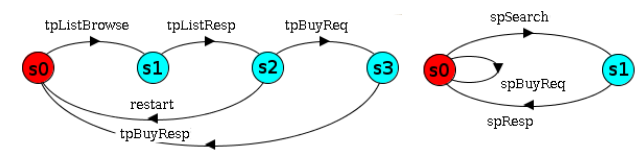

(a) Consumer

(b) Merchant

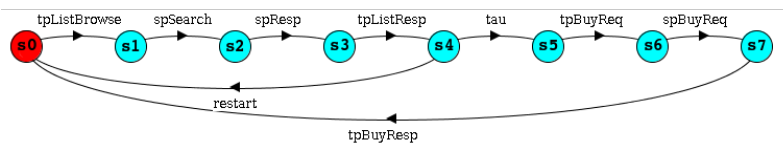

(c) Connector for one consumer and one merchant

Figure 4. LTS specifications.

of the mechanisms in the SAN model of the connected system. The ad hoc models can be parametric; for instance, a retransmission mechanism is parametric with respect to the maximum number of allowed retransmissions. As an example of mechanism and application rule, in Figure 3 we graphically show a retransmission mechanism and how to modify the original model in order to apply message retransmissions to a send operation. Specifically, the original model contains a timed activity send that models the send operation, an input gate send_cond that specifies the enabling condition of the activity, and two output gates, send_success and send_fail, that specify the output functions in the case of correct and faulty behaviour. The enhanced model is obtained from the original model by adding the following elements: a place send_count, with initial marking the maximum allowed number of retransmissions; an output gate send_count_reset, which resets the marking of send_count to its initial value when the send succeeds; an output gate send_retry, which reactivates send as long as send_count contains tokens, and resets the marking of send_count after performing all retransmission attempts.

\section{CAse Study}

In this section, we show in detail the operations performed by the modules of the Dependability unit to support the synthesis of a dependable connector in a simple case study based on a distributed marketplace with heterogeneous networked systems. In the considered scenario, consumers need to interoperate with merchants to purchase products. Consumers and merchants have heterogeneous devices. In the general case, each merchant and each consumer may use a different protocol. Without loss of generality, in this case study we assume that all merchants have the same protocol $P 1$, and that all consumers have the same protocol $P 2(P 2 \neq P 1)$.

\section{A. System Specification}

The consumer follows a tuple space protocol, e.g., LIME [15], in which communication is obtained by reading and writing tuples onto a shared space. The LIME consumer starts the interaction by writing a browse request (tpListBrowse) in the tuple space to check the availability of a certain product. After reading the response (tpListResp) from the tuple space, the consumer either restarts the interaction (restart), or writes a purchase request (tpBuyReq) and then reads the confirmation of successful transaction ( $\mathrm{pBuy}$ Resp).

The merchant follows a message passing protocol, e.g., SOAP [16]. In the SOAP protocol, the consumer starts the interaction with a discovery phase (spSearch). After receiving all responses ( $\mathrm{spResp}$ ) from the available merchants, the consumer either directly sends a purchase request to a merchant (spBuyReq), or starts a new discovery phase.

The synthesised connector is generated according to the algorithm reported in [5], and enables interaction between one consumer and many merchants. Specifically, a tpListBrowse from a consumer is translated into a multicast spSearch directed to all merchants; the spResps from the available merchants are collected and translated into a single tpListResp; a tpBuyReq from a consumer is translated into an spBuyReq followed by a tpBuyResp.

The LTSs of consumer, merchant and connector are shown in Figure 4. The LTS of the connected system is obtained as the parallel composition of the LTSs of consumers, connectors and merchants. We consider the case in which the number of consumers (merchants) ranges over $[1,100]$ $(\{1,3,5\})$. The complete FSP specification of the system is reported in [17].

Annotations: All transitions are exponentially distributed, and have a rate equal to one message per second. The probability that the consumer restarts the communication is $P_{\text {restart }}$. The failure mode of a transition associated with any send (spsearch, tpListResp, spBuyReq and tpBuyResp) and any receive (tpListBrowse, spResp, tpBuyReq) action performed by the connector is message omission. The omission probability of the transition is linked to the number of devices using the channel at the same time. Consumers and merchants use different channels, and we consider an exponential relation between the omission probability and the number of devices that use the channel. Taking into account recent works that analyse failure probability in heterogeneous scenarios, e.g., [18], we assume that the omission probability for consumers results in the interval $[0.7,0.85]$, while for merchants results in the interval $[0.92,0.96]$. 


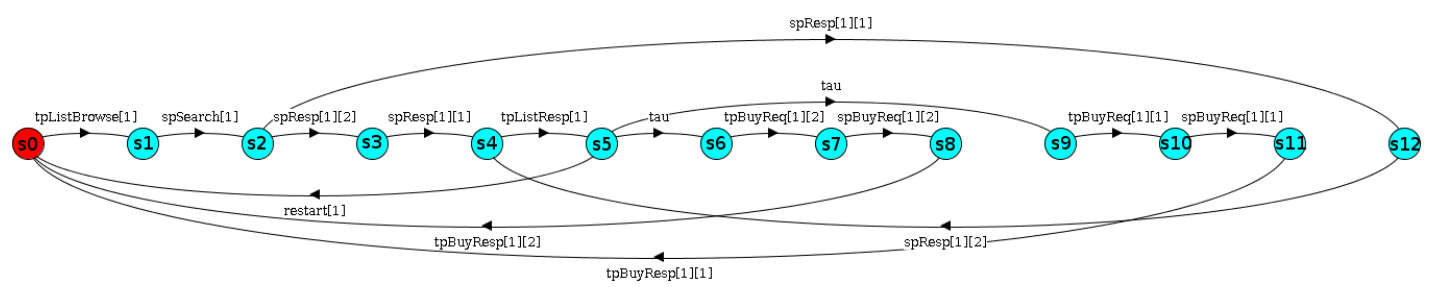

(a) LTS model of the connected system.

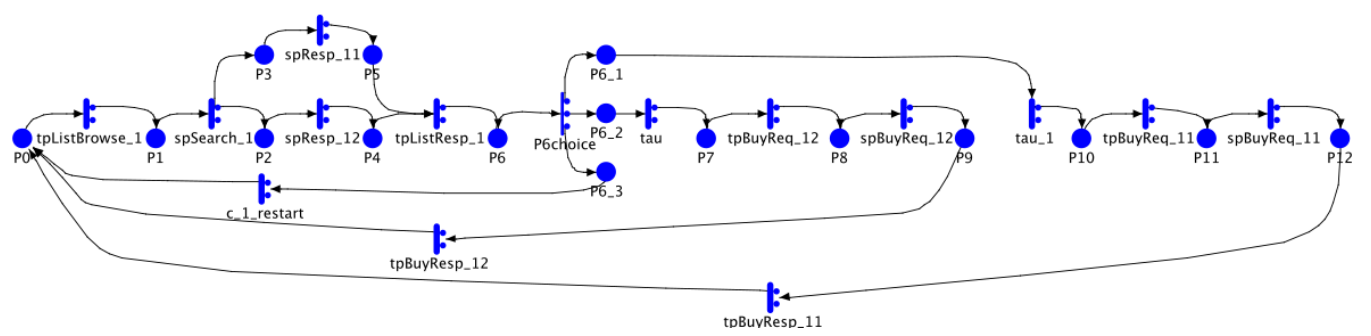

(b) SAN model of the connected system.

Figure 5. Connected system models with one consumer, one connector and two merchants

\section{B. Dependability Requirements}

The metric is Service Discovery Ratio $(S D R)$, which gives the mean probability for a consumer to successfully complete a purchase transaction with a merchant in a given time frame. The metric is specified in terms of networked system transitions as follows: $S D R(t 1, t 2)=\frac{\#((\mathrm{~S} 3, \text { tpBuyResp }, \mathrm{S} 0), t 1, t 2)}{\#((\mathrm{~S} 0, \text { tpListBrowse }, \mathrm{S} 1), t 1, t 2)}$. The guarantee on the metric is mean $(S D R(0,200)) \geq 0.7$. The constraints are: (i) the consumer performs only one, fault-free, tpListBrowse request during the time frame $[0,200]$, and (ii) a product is purchased after the consumer has successfully checked the availability of a merchant. These constraints can be expressed by the conjunction of the following conditions on the consumer LTS: the tpListBrowse request completes exactly once during the time frame $[0,200]$, i.e., $\#((\mathrm{~S} 0$, tpListBrowse, $\mathrm{S} 1), 0,200)=1$, the connector correctly receives the request, i.e., $P($ fail(S0, tpListBrowse, S1) | fire(S0, tpListBrowse, $\mathrm{S} 1))=0$, and the consumer does not restart the transaction, i.e., $P($ fire $(\mathrm{S} 2$, restart, $\mathrm{S} 0))=0$.

\section{Automated Dependability Analysis}

Dependability Model: The SAN model automatically generated by the Builder module has a timed activity for each labelled transition in the LTS. All activities have two case probabilities: case 1 is associated to the correct behaviour, while case 2 models the omission failure specified in the annotations. In order to show a human-readable model, in Figure 5(a) we show the LTS model in the case of one consumer and two merchants, and in Figure 5(b) we show the SAN model obtained from such LTS.
Reward Model: The syntax tree of the $S D R$ metric expression has two branches: the quotient operator is the root of the tree, and the dividend and divisor are the two branches of the tree. The functions of the two branches are translated into impulse reward functions associated to the timed activities tpBuyResp, and tpListBrowse. Each reward function returns 1 every time the associated transition fires. The impulse rewards are evaluated during the time frame $[0,200]$, as specified in the guarantee.

Dependability Assessment: The generated reward model is evaluated via simulation. Connected systems with different number of consumers $(c)$ and merchants $(m)$ are considered, according to the provided specification $(c \in[10,100]$ and $m \in\{1,3,5\})$. In Figure 6(a), we show the threshold specified in the requirements $(70 \%)$ and the trend of $S D R$ (on the $y$ axis) for different number of consumers (on the $x$ axis) and different number of merchants (one curve for each possible number of merchants). We can notice that $S D R$ monotonically decreases as the number of consumers gets larger. This is reasonable because, according to the specification of the channel used by the connector, larger number of consumers leads to higher message loss. The same applies when comparing the curves with different number of merchants: if we fix the number of consumers, $S D R$ is lower when the connected system contains a larger number of merchants. This is due to the fact that the connector must wait for the responses from all merchants before replying to the consumer, and the probability that at least one response gets lost increases when the system contains a larger number of merchants.

Evaluation of Results: The guarantee $(S D R \geq 0.7)$ is not satisfied for the admissible range of consumers and merchants. The Evaluator, hence, reports a warning message to Synthesis. In the following, we consider the case in which 


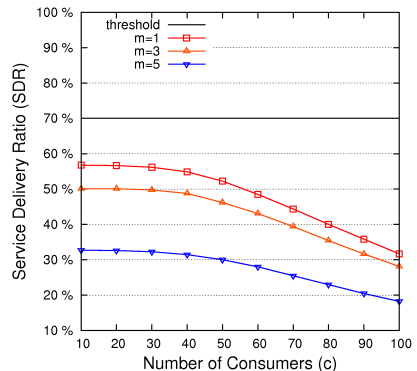

(a) Synthesised connector.

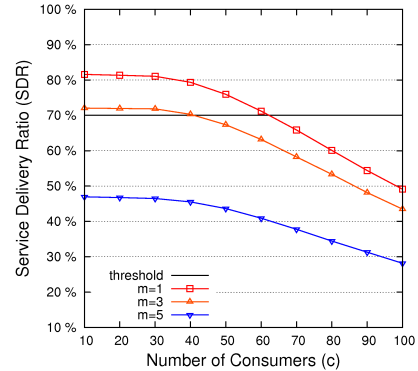

(b) Alternative deployment.
Figure 6. Metric assessment.

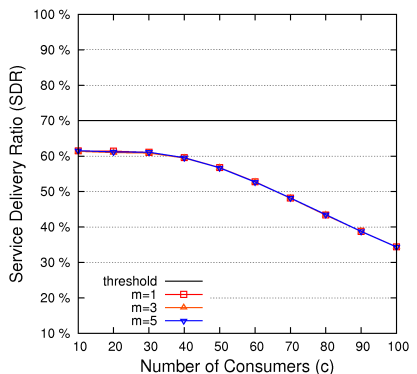

(a) Failures on consumers' channel.

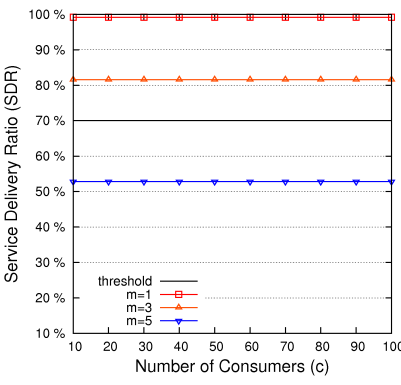

(b) Failures on merchants' channel.
Figure 7. Sensitivity analysis.

Synthesis replies with a combined improvement request with a new deployment type and a dependability mechanism, which requires the evaluation of both improvements. The specification associated with the new deployment reports that the failure mode on the consumers' channel is still linked to the number of devices that use the channel through an exponential relation, but that the omission probability results in the range $[0.81,0.96]$. The first improvement to be evaluated is a new deployment type.

Dependability Enhancement: The analysis results for the alternative deployment are shown in Figure 6(b). The guarantee is still not met for connected systems with high number of consumers and merchants.

The Evaluator, hence, triggers a sensitivity analysis for the possible failure modes. The impact of each failure mode is evaluated separately. The results of the analysis when considering only the omission failure on the consumers' channel are shown in Figure 7(a); the results when considering only the omission failure on the merchants' channel are shown in Figure 7(b). The Evaluator gives the highest rank to the failure mode on the consumers' channel because the number of times the guarantee is dissatisfied is higher when the failure on the consumers' channel is enabled.

The Enhancer, according to the considered ontology of dependability mechanisms, chooses a message retransmission technique to contrast the omission failures. We assume that the ontology reports that the maximum allowed number of retransmissions is 2. Figure 8(a) shows the analysis results for a connected system when the connector is enhanced with
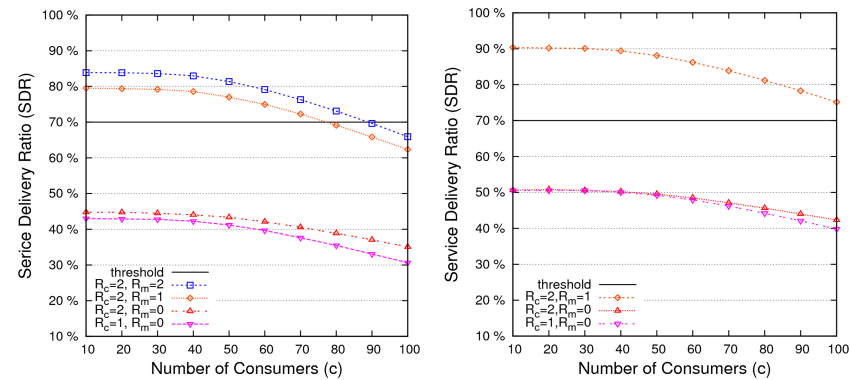

(a) Original deployment with re- (b) Alternative deployment with retransmissions.

transmissions.

Figure 8. Metric assessment when using dependability mechanisms

message retransmissions. The figure reports the value of $S D R$ in the case of 5 merchants, which is the most critical scenario. In the figure, $R_{c}\left(R_{m}\right)$ represent the number of retransmissions performed on the consumers' (merchants') channel. The Enhancer tries to apply first the retransmission mechanism to the transitions performed on the consumers' channel. Nevertheless, the guarantee is not satisfied even when the maximum allowed number of retransmissions is used. Hence, the Enhancer applies the retransmission technique also to the transitions performed on the merchants' channel. Although $S D R$ gets further improved, the guarantee is still not satisfied when the connected system contains high number of merchants.

The Evaluator, then, triggers an analysis of the connected system in the case of combined use of the two improvements. Figure 8(b) shows the analysis results for a connected system with 5 merchants (the most critical scenario): the first combination found by the Evaluator that allows to satisfy the guarantee is the alternative connector deployment with two retransmissions on the consumers' channel $\left(R_{c}=2\right)$ and one retransmission on the merchants' channel $\left(R_{m}=1\right)$. This information is reported to Synthesis in order to enable the deployment of an enhanced connector that satisfies the given dependability requirements.

\section{Discussion AND RELATED WORK}

The architectural structure and implementation described in this paper constitute an important step towards the definition of an automated procedure to provide dependability analysis as a support for the synthesis of dependable connectors. There are various aspects that still need to be investigated to fully reach the ambitious goal of automated dependability analysis, including (i) how to optimise the analysis in order to respect given time constraints, (ii) compositional solution methods for the dependability model when the synthesised connector is derived as specialisation of an already analysed connector, (iii) the definition of an ontology to support the identification of dependability mechanisms suitable to enhance the dependability level of the synthesised connector. 
Research on the definition of transformation-based verification and validation environments are being pursued since several years. Providing automatic/automated transformations methods from system specification languages to modelling languages amenable to perform dependability analysis has been recognised as an important support for improving the quality of systems. In addition, it favours the application of verification and validation techniques at industry level, where these methods are not widely used primarily due to the high level of abstractness of the mathematical modelling and analysis techniques. To provide some examples of related work on automated dependability analysis, in [19], the Viatra tool is presented, which allows to automatically check consistency, completeness, and dependability requirements of systems designed using the Unified Modelling Language; in [20], an autonomic architecture is shown that uses Markov-chain quantitative analysis to dynamically adjust the parameters of an IT system.

\section{CONCLUSiOnS}

This paper has presented an approach for the realisation of a completely automated dependability analysis in heterogeneous and dynamic environments. The presented research activity is undertaken in the context of the framework proposed in the European project CONNECT, which is general and well suited for addressing interoperability in the context of complex pervasive systems. A modelbased approach has been adopted, which can be successfully employed since the early stages of the connector synthesis. The logical architecture of a Dependability unit that supports the synthesis of dependable connectors has been presented together with a prototype implementation. A simple case study has been considered to show how the approach works and the utility of the Dependability unit in guiding the connector synthesis.

\section{ACKNOWLEDGEMENTS}

This work is partially supported by the EU FP7 Project CONNECT (FP7-231167).

\section{REFERENCES}

[1] M. Weiser, "The computer for the twenty-first century," Scientific American, pp. 94-104, September 1991.

[2] "EU FP7 Project Connect (FP7-231167)," 2009-2013.

[3] A. Avizienis, J.-C. Laprie, B. Randell, and C. Landwehr, "Basic concepts and taxonomy of dependable and secure computing," IEEE Transactions on Dependable and Secure Computing, vol. 1, pp. 11-33, 2004.

[4] "Mobius tool," http://www.mobius.illinois.edu/.

[5] R. Spalazzese and P. Inverardi, "Mediating connector patterns for components interoperability," in Proceedings of ECSA2010, LNCS 6285, 2010, pp. 335-343.
[6] H. Raffelt, B. Steffen, and T. Berg, "Learnlib: a library for automata learning and experimentation," in FMICS '05. New York, NY, USA: ACM, 2005, pp. 62-71.

[7] J. Magee and J. Kramer, Concurrency: state models \& Java programs. New York, NY, USA: John Wiley \& Sons, 2006.

[8] A. Movaghar and J. F. Meyer, "Performability modelling with stochastic activity networks," in 1984 Real-Time Systems Symposium. Austin, TX: IEEE Computer Society Press, December 1984, pp. 215-224.

[9] W. H. Sanders and J. F. Meyer, "Stochastic activity networks: formal definitions and concepts," pp. 315-343, 2002.

[10] A. Ehrenfeucht and G. Rozenberg, "Partial (set) 2-structures. Part I: basic notions and the representation problem," Acta Inf., vol. 27, no. 4, pp. 315-342, 1990.

[11] J. Cortadella, M. Kishinevsky, L. Lavagno, and A. Yakovlev, "Deriving petri nets from finite transition systems," IEEE Transactions on Computers, vol. 47, no. 8, pp. 859-882, 1998.

[12] U. Buy and G. Singal, "Toward efficient algorithms for generating compact petri nets from labeled transition systems,' in COMPSAC '02. Washington, DC, USA: IEEE Computer Society, 2002, pp. 717-722.

[13] J. Carmona, J. Cortadella, and M. Kishinevsky, "Genet: A tool for the synthesis and mining of petri nets,' in ACSD '09. Washington, DC, USA: IEEE Computer Society, 2009, pp. $181-185$.

[14] ReSIST Consortium, "EU project ReSIST: Resilience for Survivability in IST. Deliverable D33: Resilience-explicit computing.” Tech. Rep., 2008, http://www.resist-noe.org/.

[15] A. L. Murphy, G. P. Picco, and G.-C. Roman, "Lime: A coordination model and middleware supporting mobility of hosts and agents," ACM Trans. Softw. Eng. Methodol., vol. 15, no. 3, pp. 279-328, 2006.

[16] M. Gudgin, M. Hadley, N. Mendelsohn, J.-J. Moreau, and H. F. Nielsen, "Soap version 1.2 part 2: Adjuncts," W3C Recommendation, June 2003. [Online]. Available: http://www.w3.org/TR/soap12-part2/

[17] P. Masci, M. Martinucci, and F. Di Giandomenico, "Towards automated dependability analysis of dinamically CONNECTed systems," ISTI-CNR, Tech. Rep., 2010, 2010-TR-022.

[18] P. Masci, S. Chiaradonna, and F. Di Giandomenico, "Dependability analysis of diffusion protocols in wireless networks with heterogeneous node capabilities," in EDCC2010. IEEE Computer Society, 2010, pp. 145-154.

[19] G. Csertan, G. Huszerl, I. Majzik, Z. Pap, A. Pataricza, D. Varro, and D. Varr, "VIATRA - Visual Automated Transformations for Formal Verification and Validation of UML Models," in ASE'02, 2002, pp. 267-270.

[20] R. Calinescu and M. Kwiatkowska, "Using quantitative analysis to implement autonomic IT systems," in ICSE '09. Washington, DC, USA: IEEE Computer Society, 2009, pp. $100-110$. 